\title{
Les techniques d'imagerie 3D au service de la valorisation scientifiques des collections anatomiques
}

3D imagery techniques available for scientific improvement of anatomical collections

Marc Herbin, Vincent Dupret, Florent Goussard et Gaël Clément

\section{OpenEdition}

\section{Journals}

Édition électronique

URL : http://journals.openedition.org/ocim/105

DOI : 10.4000/ocim.105

ISSN : 2108-646X

Éditeur

OCIM

Édition imprimée

Date de publication : 1 septembre 2010

Pagination : 13-18

ISSN : 0994-1908

\section{Référence électronique}

Marc Herbin, Vincent Dupret, Florent Goussard et Gaël Clément, « Les techniques d'imagerie 3D au service de la valorisation scientifiques des collections anatomiques », La Lettre de I'OCIM [En ligne], 131 | 2010, mis en ligne le 01 septembre 2012, consulté le 01 mai 2019. URL : http:// journals.openedition.org/ocim/105; DOI : 10.4000/ocim.105 


\section{Une alternative à la dissection pour l'étude morphologique de spécimens rares}

Le Muséum national d'Histoire naturelle (MNHN, Paris) possède une importante collection de pièces anatomiques en fluides (la plupart étant conservées dans une solution formolée à $6 \%$ ) dont le nombre est actuellement évalué à une dizaine de milliers de spécimens. Cette collection est constituée de spécimens entiers ou d'organes isolés de vertébrés, collectés depuis le début du XIXe siècle. Parmi les spécimens les plus rares, le MNHN de Paris peut se targuer de loger la collection de cœelacanthes la plus complète au monde, avec une trentaine de spécimens.

Latimeria, l'unique genre actuel de cœlacanthe, est considéré comme le plus basal représentant actuel du groupe des sarcoptérygiens (comprenant également les dipneustes et l'ensemble des vertébrés terrestres actuels). À ce titre, le cœlacanthe est considéré comme notre très lointain « cousin » poisson. Alors que les cœlacanthes étaient supposés ne pas avoir survécu à la crise du Crétacé-Tertiaire (-70 millions d'années), un coelacanthe actuel fut signalé en Afrique du Sud par Miss Courtenay-Latimer en 1938. Après 14 années de recherches intensives, un deuxième spécimen fut pêché au large des Comores. Puis d'autres spécimens y furent bientôt capturés, révélant ainsi l'une des principales populations de cœlacanthes.

Le monopole français sur la capture et l'étude des cœlacanthes des Comores, françaises jusqu'en 1975, a permis de concentrer les spécimens au laboratoire d'Anatomie comparée du MNHN de Paris. C'est à partir des spécimens de cette collection que, indépendamment d'une centaine d'articles, trois ouvrages complets traitant de l'anatomie de Latimeria chalumnae ont été remarquablement rédigés et illustrés par J. Millot, J. Anthony et D. Robineau, alors chercheurs dans ce laboratoire. Le cœlacanthe est ainsi l'un des poissons dont l'anatomie a été la plus étudiée et, paradoxalement, l'un des poissons les plus rares (sa population actuelle est estimée à quelques centaines d'individus localisés essentiellement aux Comores, en Tanzanie, et en Indonésie, avec quelques rares signalements au large du Mozambique, de Madagascar et de l'Afrique du Sud). Par la suite, la majorité des études s'est concentrée sur la physiologie des colacanthes. Ces études (histologie, biochimie...) ont nécessité de très nombreux prélèvements sur les spécimens disponibles dans les collections. Mais les années passant, et les prélèvements aidant, cette impressionnante collection ne présente plus à l'heure actuelle que de très rares spécimens complets. Ainsi seuls quatre cœlacanthes (trois adultes et un juvénile pré-parturition) apparaissent être les derniers spécimens absolument intacts (non éviscérés, non éventrés) conservés en formol au MNHN de Paris. Quelques rares autres spécimens complets sont encore visibles dans certains musées de France (moins d'une demi-douzaine répertoriés à ce jour).

Les cœlacanthes ont été, et sont toujours, extrêmement convoités par les musées du monde. Les rares collections (publiques et privées) en possédant ont bien entendu tout l'intérêt de la communauté scientifique.

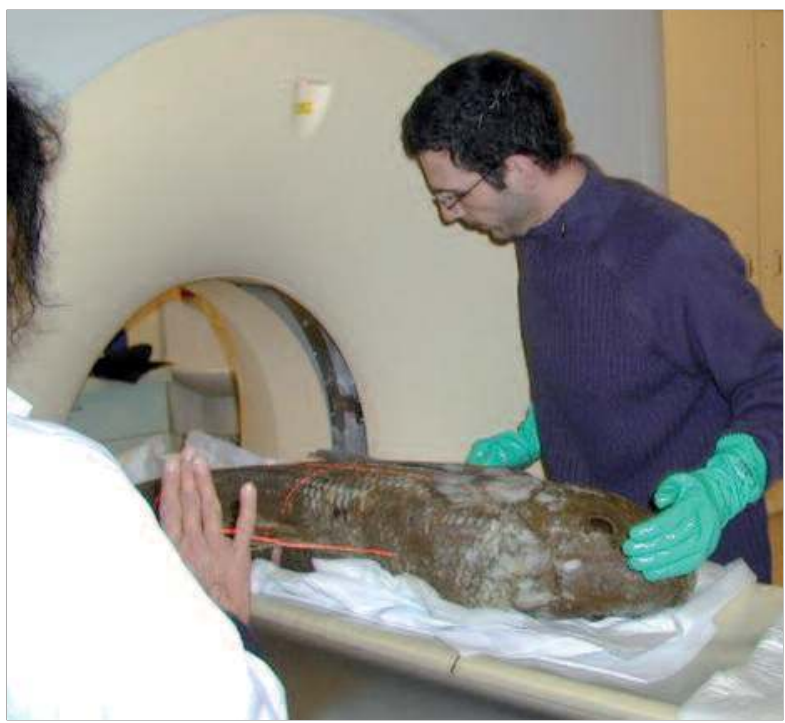

Avant son passage au scanner, le cœlacanthe est positionné sur le flan droit sur la table du CT-scan. Le spécimen a été préalablement transféré pendant un mois dans un caisson d'eau claire, afin de limiter au maximum les émanations de formol dans la salle de scanner. Une bâche de protection a été disposée sur la table pour la protéger des écoulements de liquide et de graisse suintants de l'animal. () P. Clément

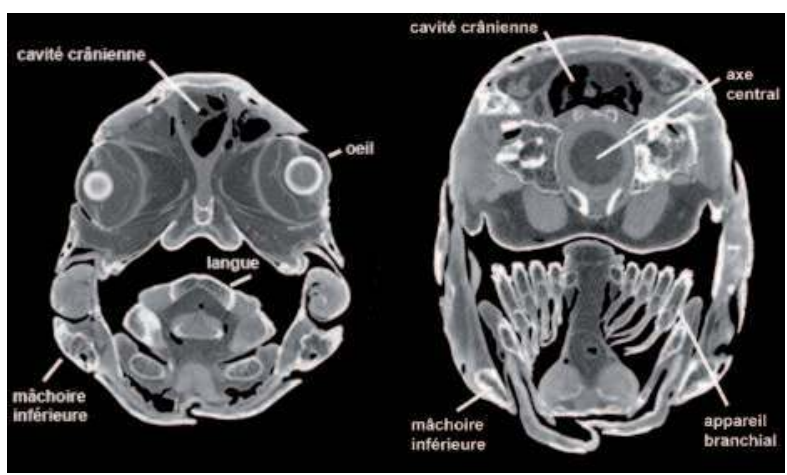

Scanner du Latimeria chalumnae (n॰ MNHN C20). Images des coupes transversales au niveau des globes oculaires et au niveau de l'extrémité postérieure de la mâchoire inférieure. Sur chacune des coupes, les différentes parties anatomiques sont facilement identifiables. (c) G. Clément/UMR7207 
Si environ 300 cœlacanthes ont été officiellement recensés (communication personnelle de R. Nulens et L. Scott du South African Institute for Aquatic Biodiversity de Grahamstown, Afrique du Sud, chargés de l'inventaire des cœlacanthes péchés), nous ne pouvons qu'estimer le nombre réel de spécimens capturés (il existe malheureusement un marché parallèle très lucratif de vente de cœlacanthes). Des mesures de protection ont donc été prises dans l'espoir de préserver les populations subsistantes. Malheureusement la loi de protection n'empêche pas les prises accidentelles liées aux méthodes de pêche intensive. Ainsi, une quarantaine de captures ont été enregistrées dans les eaux tanzaniennes depuis 2003.

Les études anatomiques des cœlacanthes, nécessitant dissections et prélèvements de tissus, ne doivent donc plus désormais se restreindre qu'aux spécimens conservés dans les collections mondiales. Mais ce stock déjà bien entamé n'est pas éternel. Si les études moléculaires, physiologiques et histologiques auront toujours besoin d'échantillons de fluides ou de tissus (généralement en petite quantité), qu'en sera t-il des études morphologiques? Il n'est plus raisonnable de disséquer des cœlacanthes pour des études de morphologie descriptive qui sont cependant toujours nécessaires. Les spécimens complets en collections sont en effet désormais beaucoup trop rares et aucune récolte de cœlacanthes, même à but scientifique, n'est heureusement plus autorisée.

Si ce problème restait entier jusqu'à très récemment, les progrès technologiques en imagerie médicale et la puissance des nouveaux ordinateurs permettent aujourd'hui de réaliser des études anatomiques virtuelles du cœlacanthe. Pour être efficace, cette alternative à la dissection nécessite que le spécimen virtuel soit aussi informatif que le spécimen original, c'est-à-dire qu'il présente les détails anatomiques les plus fins et qu'il puisse être aisément visualisé et manipulé virtuellement au sein du corps ou isolé.

\section{L'observation et l'étude par rayons $X$ et IRM}

Un cœlacanthe adulte mâle de $35 \mathrm{~kg}$ et de $130 \mathrm{~cm}$ ( ${ }^{\circ}$ MNHN C20), pêché aux larges des Comores en 1960 et conservé depuis dans une solution de formol à $7 \%$, a été utilisé pour réaliser ces études anatomiques virtuelles.
En partenariat avec le service de Radiologie et d'Imagerie médicale d'un centre hospitalier d'Île-deFrance, ce spécimen a été scanné par tomographie par rayons $\mathrm{X}$ assistée par ordinateur (technique communément appelée CAT scan pour « Computed Axial Tomography scanning »). En radiologie, les rayons X traversent de manière différentielle les structures constitutives du corps et impressionnent un film, mettant ainsi en valeur les structures internes en fonction de leur densité : les os denses apparaissent à
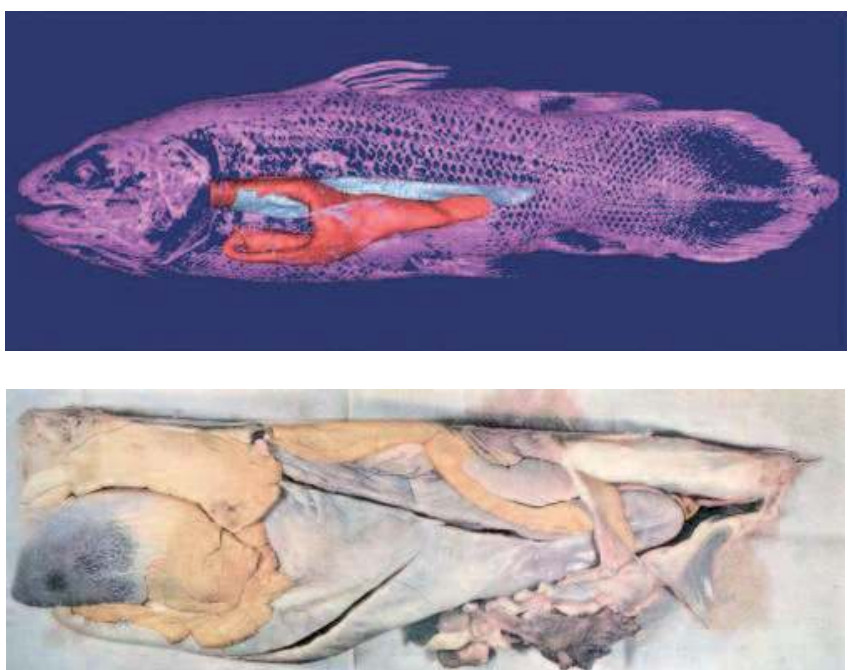

Modélisation du Latimeria chalumnae ( $n^{\circ}$ MNHN C20). En haut, la modélisation en 3D de la totalité de la surface externe du spécimen et visualisation d'une partie de son système digestif (Materialise logiciel Mimics version 12.1).

En bas, la masse viscérale correspondant aux mêmes structures extraite en 1974 d'un cœlacanthe fraîchement fixé ( $n^{\circ} \mathrm{MNHN}$ C6).

(c) G. Clément/UMR7207 et MNHN/Laboratoire d'Anatomie comparée
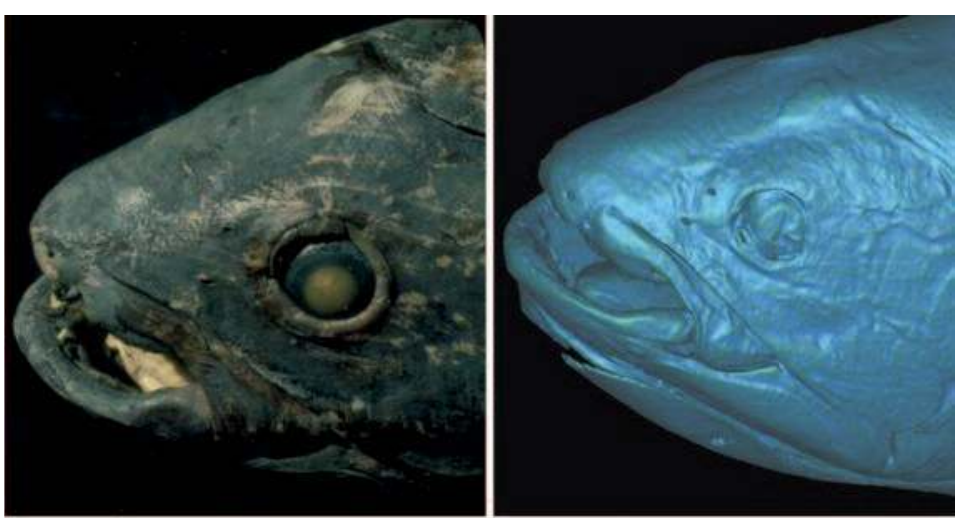

Modélisation du Latimeria chalumnae ( ${ }^{\circ}$ MNHN C20). Photographie de la tête d'un spécimen fraîchement capturé et reconstruction en 3D de la tête du spécimen $n^{\circ}$ MNHN C20.

Ce résultat peut être aussi obtenu en utilisant un scanner surfacique 3D (Materialise logiciel Mimics version 12.1). (c) MNHN/Laboratoire d'Anatomie comparée et V. Dupret/UMR7207

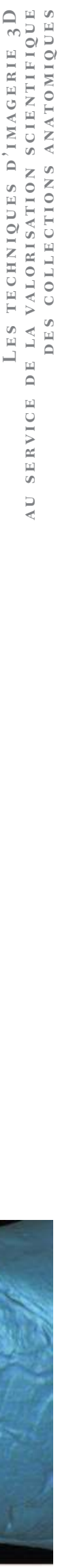

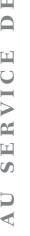


l'image plus lumineux que les cartilages, eux-mêmes apparaissant plus lumineux que les tissus mous.

Le grand avantage de la tomographie médicale repose sur le mouvement de la source de rayons X, celle-ci tournant autour du corps allongé, et sur le grand nombre de récepteurs permettant d'enregistrer jusqu'à un millier d'images par tour. L'ordinateur traite ensuite ces données pour reconstituer des tranches virtuelles du corps, dont l'épaisseur est définie par l'opérateur. Le cœlacanthe a ainsi été virtuellement découpé en tranches de 700 microns d'épaisseur selon les trois plans (horizontal, coronal, sagittal), pour un total d'environ 3500 images, individuellement analysables.

Les avancées technologiques en modélisation tridimensionnelle permettent aujourd'hui de travailler image par image et de sélectionner les parties anatomiques ou organes à caractériser. Ce travail demande bien évidemment une certaine connaissance de l'anatomie du spécimen étudié, mais également du temps et de la patience. Dans le cadre de cette reconstruction virtuelle du colacanthe, le processus de modélisation est réalisé en collaboration entre le centre de Biologie évolutive des Organismes de l'université d'Uppsala (Suède) et le département Histoire de la Terre du MNHN de Paris (UMR 7207 du CNRS/MNHN).

Chaque organe ou chaque complexe anatomique est détouré, puis modélisé individuellement. Ainsi, on pourra choisir de disséquer virtuellement une nageoire pectorale qui pourra d'abord apparaître dans son intégralité à l'écran, puis un clic de souris permettra d'ôter les écailles pour faire apparaitre les muscles, puis un autre clic les fera disparaitre pour laisser apprécier le squelette. De cette façon, il sera possible de mettre en évidence et d'illustrer les différentes étapes d'acquisitions de caractères qui ont eu lieu au cours de la transition nageoire-patte en comparant ces données avec celles d'autres taxons-clés actuels (dipneustes, salamandres...) et fossiles tels que le poisson tétrapodomorphe Tiktaalik et les premiers tétrapodes dévoniens. Chaque pièce squelettique constituant la nageoire pourra ainsi être isolée, redimensionnée, et abordée selon différents angles de l'espace. Sa position par rapport aux muscles ou à l'ensemble du corps peut facilement être visualisée. Il est tout aussi aisé de manipuler d'autres parties anatomiques comme le cerveau, le foie, ou l'estomac. Ce cœlacanthe virtuel, qu'il sera ainsi possible de disséquer à loisir sur un ordinateur, évite toute atteinte au spécimen original, et la collection ne souffre donc plus de prélèvements dommageables.
La technique d'imagerie par tomographie médicale peut être remplacée ou complétée par d'autres appareillages médicaux ou industriels. Le microtomographe de laboratoire fonctionne selon le même principe que le tomographe médical, mais il permet d'obtenir une plus haute résolution (quelques microns) sur des spécimens de petites tailles (centimétriques à décimétriques). Les microtomographes de laboratoire sont utilisables sur du matériel actuel, et également fossile. Le faisceau de rayons $\mathrm{X}$ émis dans ces appareillages est en effet suffisamment puissant pour traverser des structures très minéralisées, tel qu'un fossile inclus dans la roche. Les structures internes constituant le fossile (os, coquilles, dents...) sont ainsi observables en coupe et modélisables, si besoin est, en trois dimensions. Comme exemple, cette technique a été utilisée sur un excrément fossile (coprolithe) de coelacanthe du crétacé (environ -100 millions d'années) d'Angleterre afin de visualiser la structure et les séquences de dépôt des matières fécales et tenter de déterminer la nature des proies ingérées. De petits restes osseux et des empreintes de cristallin de poissons ont été révélés au sein de ce coprolithe.

L'imagerie par résonance magnétique (IRM) se révèle également très utile. Le principe physique de l'IRM (l'observation de la réponse - la résonance magnétique nucléaire - de noyaux ciblés soumis à un champ magnétique extérieur et à une excitation électromagnétique) permet l'observation de tissus mous peu différenciés (comme les structures du cerveau) grâce à une excellente résolution en contraste.

La collection de cœlacanthes du MNHN contient également un embryon à un stade de développement avancé. Ce juvénile pré-parturition a été découvert

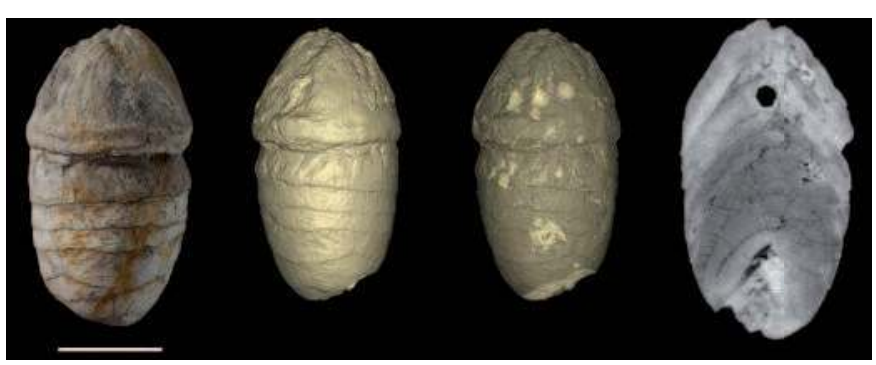

Images issues d'un microtomographe conventionnel par rayons

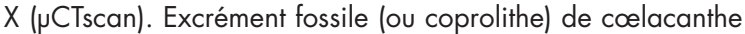
du Crétacé. De gauche à droite, photographie du spécimen (échelle $1 \mathrm{~cm}$ ), reconstruction en 3D, visualisation des structures internes et coupe longitudinale virtuelle présentant la structure en couches et quelques restes alimentaires (cristallins et débris osseux) (Materialise logiciel Mimics version 12.1). () P. Loubry/UMR7207 et G. Clément/UMR7207 
en 1975, avec quatre autres de ses frères et sœurs, dans l'oviducte d'une grande femelle $\left(\mathrm{n}^{\circ} \mathrm{MNHN}\right.$ C26), pêchée en 1962 aux Comores. Les colacanthes, ovovivipares, donnent naissance à des juvéniles de 35 à $40 \mathrm{~cm}$ de long, répliques miniatures de leurs parents. Les tentatives pour imager ce spécimen par scanner à rayons $\mathrm{X}$ (scanner médical et microtomographe conventionnel) ont toutes échoué, les coupes virtuelles ne permettant pas de correctement distinguer les structures anatomiques internes. Les densités des différents tissus (muscles, cartilages, os...) des embryons sont trop peu différenciées et sont toutes d'une valeur avoisinant celle de la solution formolée imprégnant l'ensemble du spécimen.

Dans ce contexte particulier, une autre technique d'imagerie peut être envisagée à savoir l'imagerie en résonance magnétique (IRM). Des acquisitions IRM ont donc été réalisées sur ce même embryon, avec des résultats beaucoup plus significatifs. Deux types d'acquisition ont été réalisés, l'un pour mettre en évidence les cartilages et l'autre les muscles. Les temps d'acquisition sont par contre beaucoup plus longs (plusieurs heures au lieu de quelques minutes) et la résolution généralement plus basse que par scanner à rayons $\mathrm{X}$. Les deux types d'acquisition (scanner et IRM) délivrent des suites d'images représentant les coupes virtuelles de l'animal, qui peuvent être traitées par les mêmes logiciels de segmentation (tels que Mimics, Osirix, Aviso, et VG Studio Max par exemple).

Il est ainsi possible de combiner des images issues d'un scanner par rayons $\mathrm{X}$ (révélant aisément les structures les plus minéralisées telles que le squelette osseux ou les dents) avec des images du même animal, et à la même résolution, issues d'acquisition IRM (mettant en évidence les tissus mous tels que muscles ou système nerveux).

Dans le cadre de ces études de complémentarité de sources d'acquisition tomographique, le colacanthe adulte MNHN C20 va être prochainement imagé par IRM dans une institution médicale de la région parisienne afin de préciser la morphologie de certaines structures peu apparentes sur les images obtenues par scanner médical. Les structures molles de densités proches de celle de la solution formolée sont en effet parfois difficilement interprétables sur les coupes issues de tomographie par rayons X. Les acquisitions IRM de ce colacanthe adulte seront donc paramétrées pour mettre en évidence des tissus mous spécifiques tels que l'encéphale et les cartilages et muscles des nageoires paires.
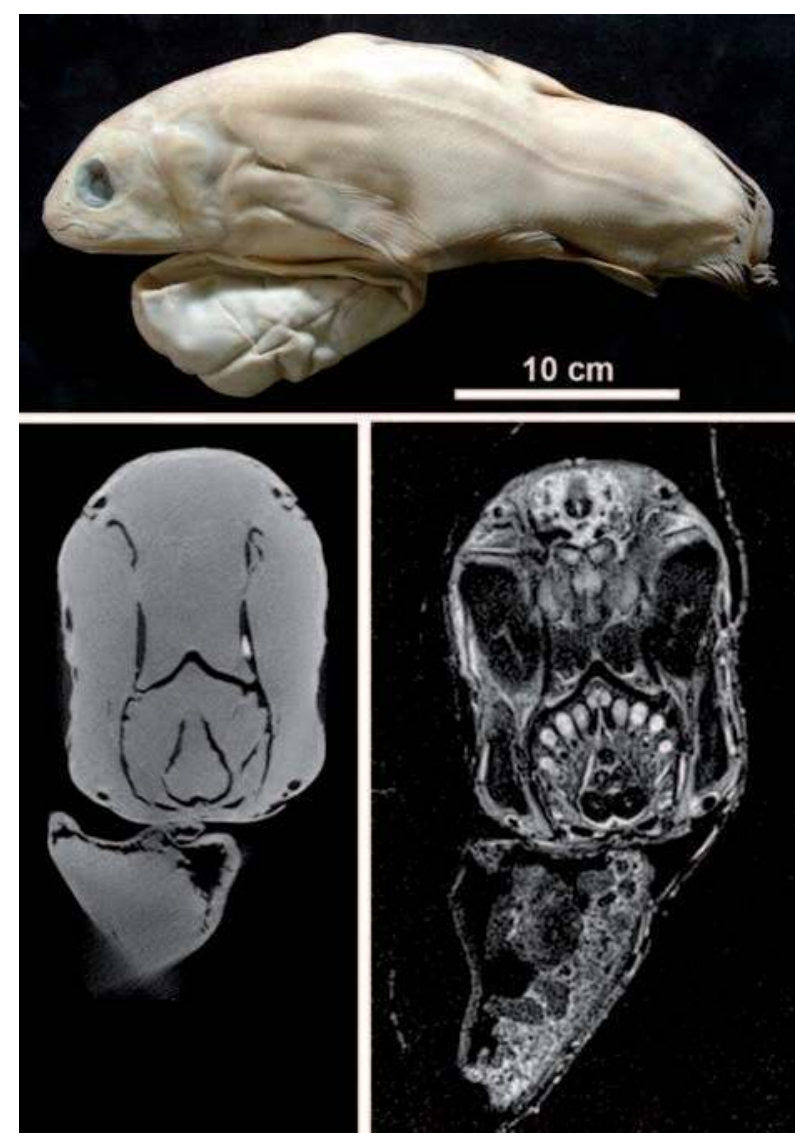

Image en Résonance Magnétique d'un embryon de cœlacanthe ( ${ }^{\circ}$ MNHN C26-5). En haut, photographie du spécimen, de gauche à droite, coupe transversale à l'arrière des yeux obtenue par un microtomographe conventionnel ( $\mu \mathrm{CTscan})$, et coupe transversale au même niveau en IRM. (c) G. Clément/UMR7207

\section{Conclusion}

Le principal intérêt de ces techniques d'investigation est d'être non invasives pour le spécimen (avec toutefois une réserve concernant l'intégrité de la structure de l'ADN), mais aussi qu'elles évitent, à long terme, de devoir renouveler les collections par le prélèvement de spécimens dans leur milieu naturel.

Il est vrai que les méthodes d'investigation par tomographie par scanner à rayons $\mathrm{X}$ et par IRM sont encore consommatrices de temps et d'argent, et d'accès parfois difficile, mais elles permettent la valorisation de spécimens de collection, jusqu'à maintenant restreints à une fonction de présentation muséologique. Elles sont donc parfaitement adaptées pour les études anatomiques de ces spécimens précieux et scientifiquement importants, ou dans le cadre d'une scénographie visant à présenter l'anatomie externe et interne d'un animal. Elles peuvent aussi être utilisées pour modéliser des spécimens 
qui ne peuvent être manipulés de façon répétitive, comme les types ou les spécimens très fragiles (entiers ou squelettes), dans une politique de conservation préventive. Les observations et les prises de mesures sont tout aussi fiables et aisées, voire facilitées, sur le spécimen virtuel que sur le spécimen original. Autre avantage muséologique, des répliques du spécimen entier, ou d'une partie du spécimen, peuvent être réalisées par la technique de prototypage (ou d'impression 3D). Les répliques, en résine, cire ou poudre consolidée, peuvent être distribuées à volonté et à toutes les échelles de taille. Ces technologies d'imagerie par rayons X et par IRM sur les spécimens, frais ou conservés en fluide, des collections d'Histoire naturelle présentent donc des intérêts significatifs dans les domaines de la recherche scientifique, de l'enseignement et de la diffusion des sciences, de la conservation préventive, et de la muséologie.

Les auteurs tiennent à remercier les responsables et les équipes des différents centres d'acquisitions par tomographie X et IRM approchés pour ces études, toujours enthousiastes à soutenir leurs recherches sur les coelacanthes. Ils sont ainsi fortement redevables à l'équipe de Radiologie et d'Imagerie médicale d'un centre hospitalier de la région parisienne; à l'équipe de recherche et développement MR Spectroscopy of the Department of Diagnostic and Interventional Radiology, Institute for Clinical and Experimental Medicine (Prague, République Tchèque) ainsi qu'à l'entreprise VISCOM de Saint-Ouen-l'Aumône et à la société Études Recherches Matériaux de Poitiers pour les acquisitions par microtomographes conventionnels. Une partie de ce travail a été réalisée à l'atelier de tomosynthèse (responsable: D. Geffard-Kuriyama) de l'UMR 7207 (CR2P) du CNRS (MNHN). Ces études d'anatomie virtuelle du coclacanthe (Latimeria) ont été initiées en partenariat avec l'équipe de l'Evolutionary Organismal Biology Centre, Uppsala, Suède (responsable: Per Ahlberg) avec un support $d u$ Swedish Research Council. La recherche de G. Clément concernant l'histoire évolutive des coclacanthes est en partie subventionnée par le projet "Terrestrialisation » financé par les programmes «ECLIPSE II » et INSU-2009 de l'INSU/CNRS. 